\title{
Comparing Saddle, Slotted-tube and Parallel-plate Coils for Magnetic Resonance Imaging
}

\author{
D. Nespor ${ }^{1}$, K. Bartusek ${ }^{2}$, Z. Dokoupil ${ }^{2}$ \\ ${ }^{1}$ Department of Theoretical and Experimental Electrical Engineering, \\ Brno University of Technology, Kolejni 2906/4, 61200 Brno, Czech Republic \\ 2 Institute of Scientific Instruments, Academy of Sciences of the Czech Republic, \\ Kralovopolska 147, 61264 Brno, Czech Republic
}

\begin{abstract}
The paper is concerned with a comparison of the properties of RF coils of three configurations for MRI measurements on small animals. In comparison with the classical saddle coil the proposed modification of slotted-tube coil exhibits identical homogeneity of $B_{1}$ field in a larger space. The parallel-plate coil has a satisfactory homogeneity of $B_{1}$ field over the whole internal space. The signal-to-noise ratio measured for all three coils is roughly the same and is given by the magnitude of RF pre-amplifier noise. As the slotted-tube and parallel-plate coils have a lower inductance compared with the saddle coil, they can be tuned to resonance on the $200 \mathrm{MHz}$ frequency even at larger dimensions. The results show that the parallel-plate coil has very good properties for the measurement of small animals.
\end{abstract}

Keywords: MRI measurements, RF coils, homogeneity of $B_{1}$, numerical analysis.

\section{INTRODUCTION}

$\mathrm{T}$ HE MRI (magnetic resonance imaging) system, which can produce high-quality images in an arbitrary cross section of the human body, has been recognized as a new powerful technique for medical diagnosis and has gradually come to be employed in practical situations. In the MRI system, an RF probe is used to emit a uniform RF magnetic field over the human body and receive the magnetic resonance signal from the body for imaging.

Several kinds of RF probes have been developed. The transverse electromagnetic birdcage resonator is commonly used for MRI. The characteristics of the birdcage resonator, including the effects of a conducting shield, have been analyzed by using the boundary element method. In this work a multiconductor transmission line (MTL) model is developed and successfully applied to analyze a 12-element unloaded and loaded microstrip line transverse electromagnetic (TEM) resonator coil for animal studies [1]. To produce a uniform $B_{1}$ field along the coil (z-direction), a multi-section alternating impedance microstrip element was investigated in Akgun [2]. The widths of the microstrip line are varied to produce an alternating low-high impedance configuration. Simulation and experimental results are compared to microstrip resonant elements.

The slotted-tube resonator (STR) is also used for MRI applications [3]-[6]. Many efforts have been made to analyze the EM parameters of the slotted-tube resonator in order to show the properties of the probe and design an optimum structure [3], [6]. In Ben Ahmed and Feham [7], [8] rigorous analytical expressions for the EM parameters of the shielded STR with a circular cross section have been obtained using the finite element method (FEM) and method of moments (MoM) in two dimensions, but without taking into account the influence of the thickness of the STR.

A saddle RF coil is used in MR systems. Single-channel systems [9], [10] require an arrangement of coils that covers the full field of view (FOV) of the imaged object. It is an important consideration to obtain a uniform and complete image of the object.

Traditional clinical $1.5 \mathrm{~T}$ magnets are being replaced by $3 \mathrm{~T}$, while research magnets of $7 \mathrm{~T}, 8 \mathrm{~T}$, and $9.4 \mathrm{~T}$ referred to as high field magnets are being used for animal and human experiments [7]-[14]. The great appeal of high field MRI is its improved image quality and resolution [15]. The signal-to-noise ratio (SNR) correlates in approximately linear fashion with field strength, being roughly twice as large at $3 \mathrm{~T}$ as at $1.5 \mathrm{~T}[16]$.

A parallel-plate waveguide was built and used to acquire images of a healthy volunteer's leg at $3 \mathrm{~T}$ on a clinical MR imager [17]. Waveguides have been successfully used to generate magnetic resonance images at $7 \mathrm{~T}$ for whole-body systems [18]. From these results, it has been established that waveguides are only suitable for $7 \mathrm{~T}$ systems with wide bores of at least $60 \mathrm{~cm}$. This is mainly due to the cut-off frequency of the cylindrical waveguides used. To overcome this limitation, a parallel-plate waveguide was employed since its cut-off frequency depends on the separation of the plates. Zanche [19] has investigated the possibility of increasing the SNR and homogeneity of the RF magnetic field $B_{1}$, to characterize microfluidic flow with direct detection. To enable this, he used a parallel-plate RF resonator with pure phase spatial encoding.

A uniform $B_{1}$ and a strong $B_{1}$ per unit current are critical for magnetic resonance imaging. To design a modified RF probe resonator, numerical simulation in a wide-frequency band is often required. The parallel plate resonator has been well investigated in non-MR research areas [21], [22], and the magnetic field $B_{1}(\mathrm{r})$ between the parallel plates has been shown to be homogeneous [20], [24].

\section{FORMULATION OF THE PROBLEM}

The aim of this work is to modify the slotted-tube resonator [6] and propose a parallel-plate RF coil for MR microscopy and measurement of small animals or plants. 
Characteristics such as the homogeneity of the RF magnetic field and achievable signal-to-noise ratio are better compared with the classical saddle coil. Some theoretical results are compared with the measured data to confirm the validity of the present simulation.

To compare the modified slotted-tube resonator and parallel-plate RF coil with the classical saddle coil, an analysis of the RF magnetic field was performed for all the above coil configurations, using the numerical analysis. The result of the analysis was an optimum coil configuration with a minimum inhomogeneity of the field $B_{1}$, which is shown in Fig.1. For the purpose of experimental comparison, three MR probes were made with coils as per Fig.1.

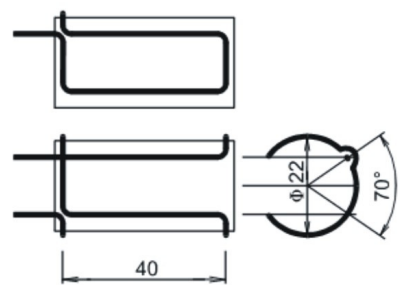

a)

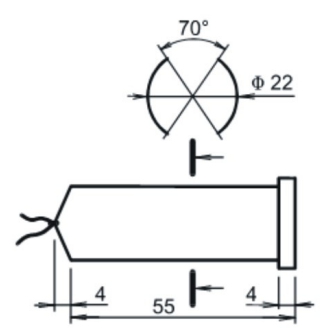

b)

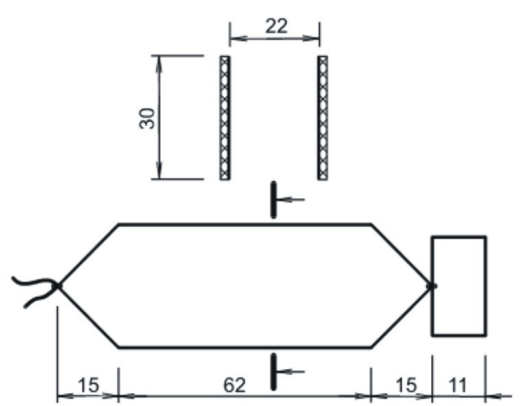

c)

Fig.1. Schematic of RF coils under examination: a) saddle coil, b) slotted-tube coil, and c) parallel-plate coil.

The saddle coil was made of silver-plated copper conductor of $1.4 \mathrm{~mm}$ in diameter. The slotted-tube and parallel-plate coils were made of silver-plated copper foil $0.05 \mathrm{~mm}$ thick. The coil angle $\Phi$ was optimized via numerical calculation to obtain minimum inhomogeneity of $B_{1}$ and is $\Phi=70^{\circ}$. This value is in agreement with the result given in [6].

For all the RF coils, the $B_{1}$ field maps were measured using the MRI methods, and compared with numerical calculation. Two main criteria were used in the comparison, namely the magnitude/size of the space of field inhomogeneity below $\Delta B_{1}<10 \%$ and $20 \%$, and the size of the signal-to-noise ratio in the image. For applications of RF coils in MR experiments in which phase MR images are used the maximum phase deviation in the image was evaluated.

\section{METHOD}

The numerical analysis of the EM field of coils was solved in two ways: first as an electromagnetostatic model and then as an RF model. The concept of magnetostatic numerical analysis has been described in [23]. The advantage of the electromagnetostatic model is its simplicity and good solution convergence in the analysis. The electromagnetostatic model can only be used in cases when the wavelength of the EM field is much larger than the coil under analysis. If the magnitude of the EM field wavelength comes close to the coil dimensions, the numerical analysis results will no longer correspond to the actual EM field distribution.
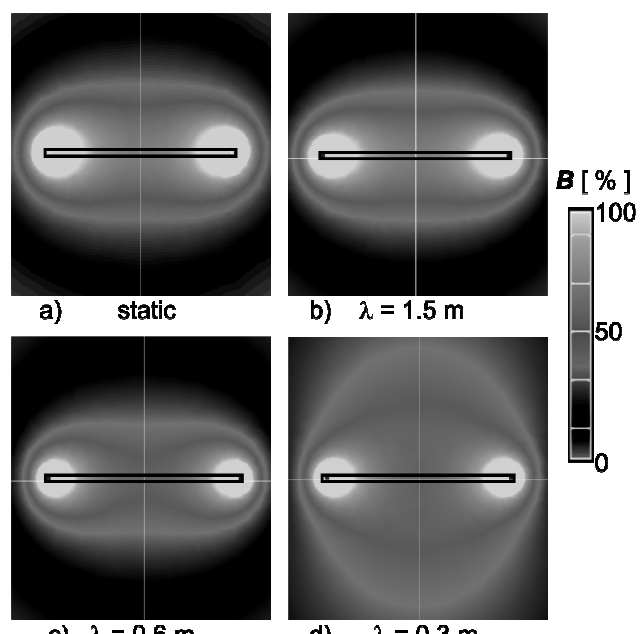

c) $\lambda=0.6 \mathrm{~m}$

d) $\lambda=0.3 \mathrm{~m}$

Fig.2. Comparison of magnetic induction distribution near circular single-turn coil with center-line length $39 \mathrm{~mm}$, calculated via EM and RF numerical analyses for different wavelengths $\lambda=1.5,0.6$ and $0.3 \mathrm{~m}$.

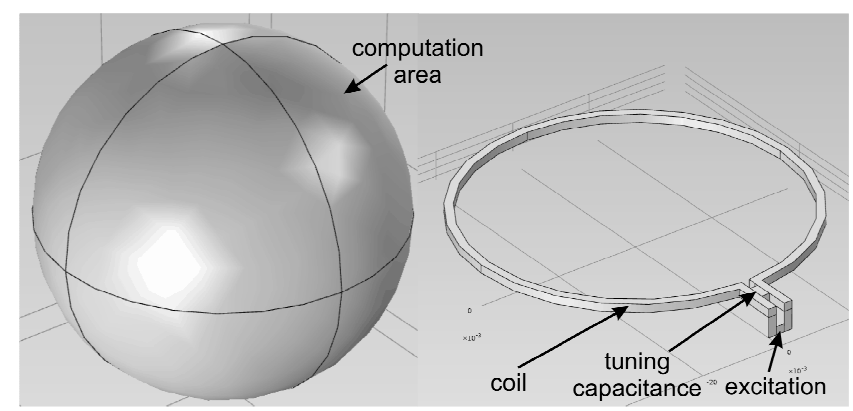

Fig.3. Geometry of numerical RF analysis of planar single-turn coil.

Fig.2. gives the distribution of magnetic induction $B$ for the circular single-turn coil of center-line length $39 \mathrm{~mm}$. The distribution of magnetic induction is shown in a crosssection perpendicular to the coil plane. Fig.2. a) shows 
magnetic induction distribution solved using the magnetostatic model. Figs.2. b), c) and d) show magnetic induction distribution solved using the RF model for different wavelengths. It is obvious from Fig.2. that for the wavelength $\lambda-1.5 \mathrm{~m}(200 \mathrm{MHz})$ the distribution of magnetic induction matches up with the results obtained using the statistical analysis. For the wavelengths $\lambda=0.6 \mathrm{~m}$ $(500 \mathrm{MHz})$ and $\lambda=0.3 \mathrm{~m}(1 \mathrm{GHz})$, however, the distribution of magnetic inductance is different.

The geometry of numerical RF analysis of planar singleturn coil is given in Fig.3. The geometric configuration of the model is made up of a coil, which is formed by the vacuum. The boundary conditions of this coil are defined as an impedance medium and are represented by (1), where $\mu$ is the permeability, $\varepsilon$ is the permittivity, $\boldsymbol{H}$ is the magnetic field intensity, $\boldsymbol{E}$ is the electric field intensity, $\gamma$ is the electric conductivity, $\omega$ is the angular frequency, and $n$ is the normal vector.

$$
\sqrt{\frac{\mu_{0} \mu_{r}}{\varepsilon_{0} \varepsilon_{r}-j \frac{\gamma}{\omega}}} \boldsymbol{n} \times \boldsymbol{H}+\boldsymbol{E}-(\boldsymbol{n} \cdot \boldsymbol{E}) \boldsymbol{n}=0
$$

EM field excitation is provided using a port with lumped parameters. The coil is complemented with additional capacitance for tuning the resonance frequency $f_{\mathrm{r}}$ to the frequency of NMR system $f=200 \mathrm{MHz}$. The coil is placed in the computation space and this space ends in perfectly matched layers (PML). PMLs prevent reflections and thus simulate an open space.

In the first part of the RF model the Eigen-frequency analysis was used to determine the first resonance frequency $f_{\mathrm{r}}$ of the coil being modeled. Based on this analysis, the magnitude of additional capacitance was defined for tuning the resonance frequency $f_{\mathrm{r}}$ to the frequency of the NMR system. Additional capacitance was defined as elements with defined relative permittivity $\varepsilon_{\mathrm{r}}$.

When solving the Eigen-frequency analysis, equation (2) was used, where the imaginary part represents Eigenfrequency, and the real part represents attenuation.

$$
\lambda=-j \omega+\delta
$$

In the second part of the RF model the wave equation (3), where $\mathrm{k}$ is the wave number, and the Maxwell equation (4) were used in the analysis of the high-frequency electromagnetic field of the coils defined.

$$
\begin{gathered}
\nabla \times \mu_{r}^{-1}(\nabla \times \boldsymbol{E})-k_{0}^{2}\left(\varepsilon_{r}-j \frac{\gamma}{\omega \varepsilon_{0}}\right) \boldsymbol{E}=0 \\
\nabla \times \boldsymbol{E}=-j \omega \mu \boldsymbol{H}=0
\end{gathered}
$$

The map of field $B_{1}$ was measured using the spin-echo technique with the tilt angle $\alpha=45^{\circ}$. The field $B_{1}$ was calculated according to the relation

$$
B_{1}=\frac{\arcsin \left(\frac{S}{S_{0}}\right)}{\gamma \tau}
$$

Where $S$ and $S_{0}$ are the intensities in the image for tilt angles of $45^{\circ}$ and $90^{\circ}$, respectively.

\section{EXPERIMENT}

The result of calculating the map of magnetic induction $B_{1}(x, y)$ of all the coils according to Fig. 1 . and using the EM method is given in Fig.4. The maps of $B_{1}(x, y)$ are in the center of the coil $(z=0)$ in a plane perpendicular to the coil axis. Fig.5. gives the isolines of the field $B_{1}$, with the maps being normalized to the value $B_{1}$ in the center of the map. The levels of field $B_{1}$ are $0.8 B_{1}, 0.9 B_{1}, B_{1}, 1.1 B_{1}$, and 1.2 $B_{1}$.

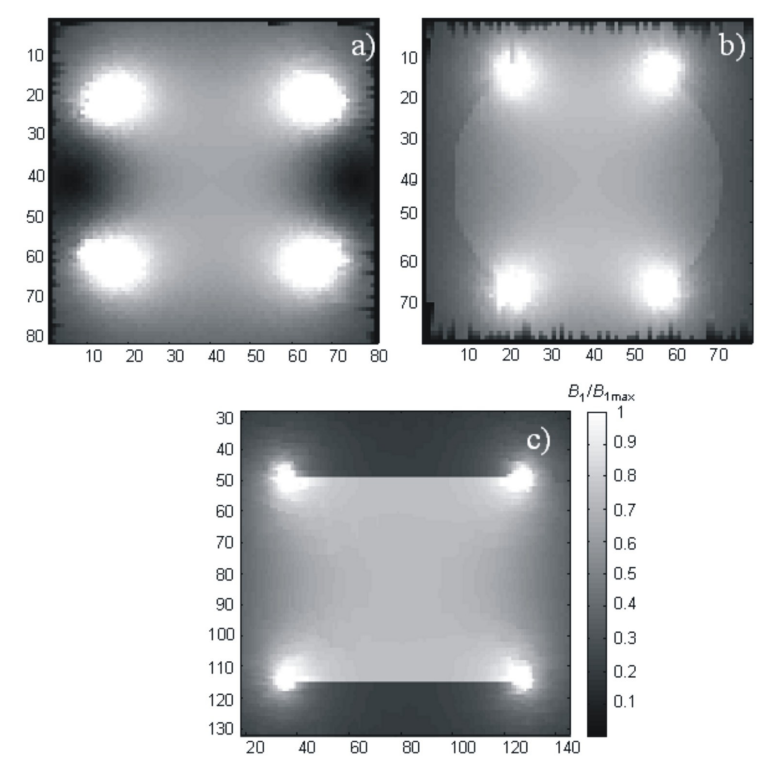

Fig.4. Map of magnetic induction $B_{1}(x, y)$ of test coils according to Fig.1. and using the EM method: a) saddle coil, b) slotted-tube coil, and c) parallel-plate coil.

The experiments were conducted on a Magnex $4.7 \mathrm{~T}$ $200 \mathrm{~mm}$ i.d. horizontal bore superconducting magnet at $200 \mathrm{MHz}$ at the Institute of Scientific Instruments. A watercooled Magnex $150 \mathrm{~mm}$ i.d. gradient set was employed, driven by IECO GPA 200-350 amplifiers, which can provide $180 \mathrm{mT} / \mathrm{m}$ maximum gradients. All experiments were performed with an MR Solution console. The data measured were processed using the MAREVISI and MATLAB programs.

One sample was measured by all the coils, which was a cylinder with a diameter of $20 \mathrm{~mm}$ and a length of $40 \mathrm{~mm}$, filled with a solution of deionized water of the following concentration: 1 liter of water, 1.2 grams of $\mathrm{NiSO}_{4}$ and 2.6 grams of $\mathrm{NaCl}$ in order to shorten the relaxation times to $T_{1}=T_{2}=130 \mathrm{~ms}$. The sample takes up the whole space of the RF coil because in order to compare the RF coils, we want to measure the map of field $B_{1}$ over the whole cross section inside the coil. 

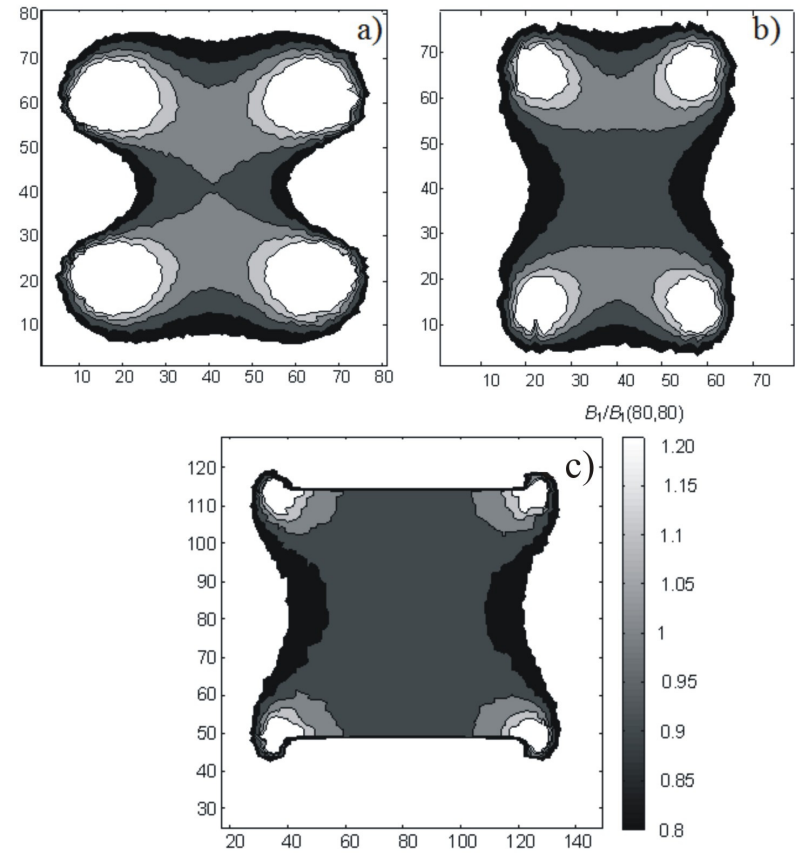

Fig.5. Simulation of the $B_{1}$ field distribution depicted as equipotential levels, a) saddle coil, b) slotted-tube coil, and c) parallel-plate coil. Field levels of $B_{1}$ are $0.8 B_{1}, 0.9 B_{1}, B_{1}$, $1.1 B_{1}$, and $1.2 B_{1}$.
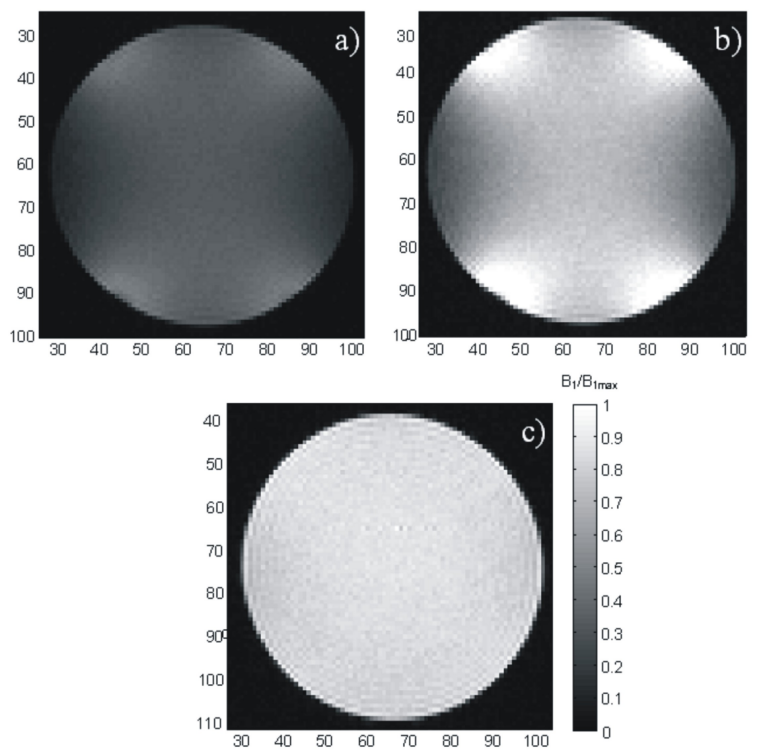

Fig.6. Map of magnetic induction $B_{1}$ in a) saddle coil, b) slottedtube coil, c) parallel-plate coil.

The spin-echo method $\left(T_{\mathrm{E}}=17 \mathrm{~ms}, T_{\mathrm{R}}=1 \mathrm{~s}\right)$ was used to measure 11 transversal slices $2 \mathrm{~mm}$ thick and $5 \mathrm{~mm}$ apart from each other. The FOV size/magnitude was 30x30 mm $(128 \times 128 \mathrm{px})$. In the calculation of the map of $B_{1}$ according to (5), two images with tilt angles $\alpha=45^{\circ}$ and $90^{\circ}$ were measured. Fig.6. gives $B_{1}$ field maps for all the configurations of RF coils. Fig.7. shows the isolines of field $B_{1}$, with the field maps normalized to the value of $B_{1}$ in the center of the map. The levels of field $B_{1}$ are $0.8 B_{1}, 0.9 B_{1}$, $\boldsymbol{B}_{1}, 1.1 B_{1}$, and $1.2 B_{1}$.
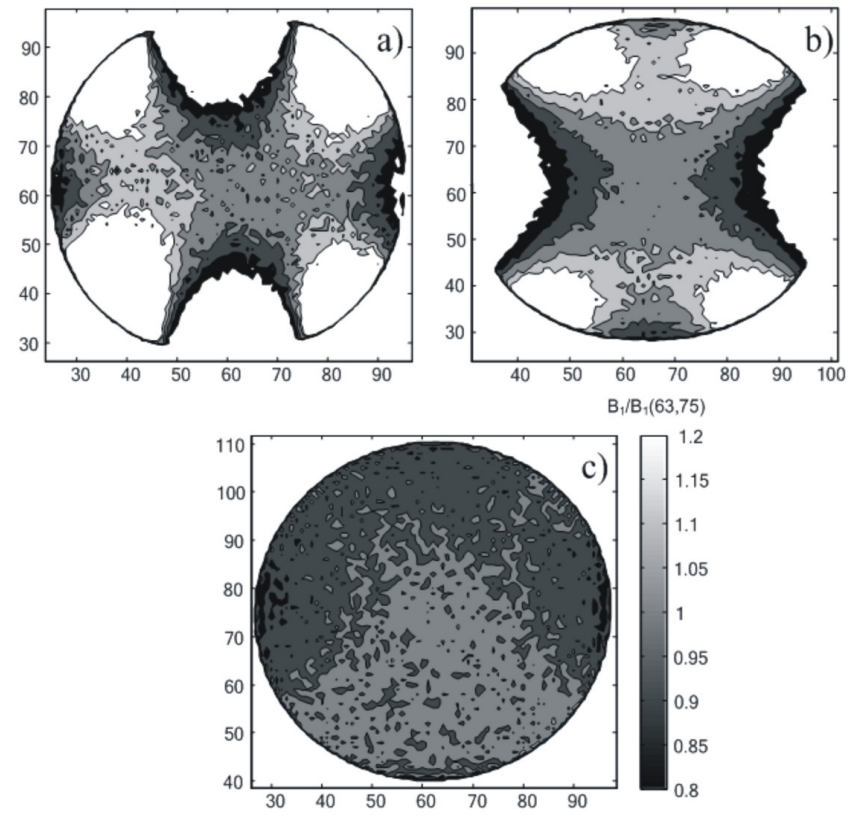

Fig.7. Equipotential levels of $B_{1}$ for: a) saddle coils, b) slotted-tube coils, c) parallel-plate coils.

Evaluated from the maps of field $B_{1}$ were the signal-tonoise ratio, size of the homogeneous circular and rectangular area for $10 \%$ increase and decrease of $B_{1}$, and for the maximum phase change in the image. The results are summarized in Table 1.

Table 1. Calculated parameters of RF coils

\begin{tabular}{|c|c|c|c|}
\hline Coil & Saddle & Slotted-tube & $\begin{array}{c}\text { Parallel- } \\
\text { plate }\end{array}$ \\
\hline $\begin{array}{c}\text { Signal to noise } \\
\text { ratio }\end{array}$ & 57.2 & 64.4 & 49.7 \\
\hline $\begin{array}{c}\text { Active coil } \\
\text { resistance } \\
(f=200 \mathrm{MHz})[\Omega]\end{array}$ & 3.22 & 0.82 & 0.51 \\
\hline $\begin{array}{c}\text { Inductance } L \\
(f=200 \mathrm{MHz}) \\
{[\mu \mathrm{H}]}\end{array}$ & 0.23 & 0.051 & 0.048 \\
\hline $\begin{array}{c}\text { Homogeneity } \\
( \pm 10 \%): \\
\text { circle with } \\
\text { diameter [mm] }\end{array}$ & 8.9 & 9.8 & 20 \\
\hline $\begin{array}{c}\text { Homogeneity } \\
\left( \pm 10^{\circ} \%\right): \\
\text { rectangle } \\
\text { dimensions }[\mathrm{mm}]\end{array}$ & $17.7 \times 4.5$ & $11.5 \times 10.4$ & $20 \times 20$ \\
\hline \begin{tabular}{c}
$\left.\Delta \Phi{ }^{0}\right]$ \\
\hline
\end{tabular} & 7.4 & 9.8 & 3.5 \\
\hline
\end{tabular}

Using the parallel-plate coil the spin-density MR images of biological tissues (chicken wing and euphorbia) taking up the whole space within the coil were measured. The SE method was used, with echo time $T_{\mathrm{E}}=17 \mathrm{~ms}$, repeat time $T_{\mathrm{R}}=3 \mathrm{~s}$, slice thickness $3 \mathrm{~mm}$, field of view $40 \times 40 \mathrm{~mm}, 256 \times 256 \mathrm{px}$ for the chicken wing and $30 \times 30 \mathrm{~mm}, 128 \times 128$ px for euphorbia. 

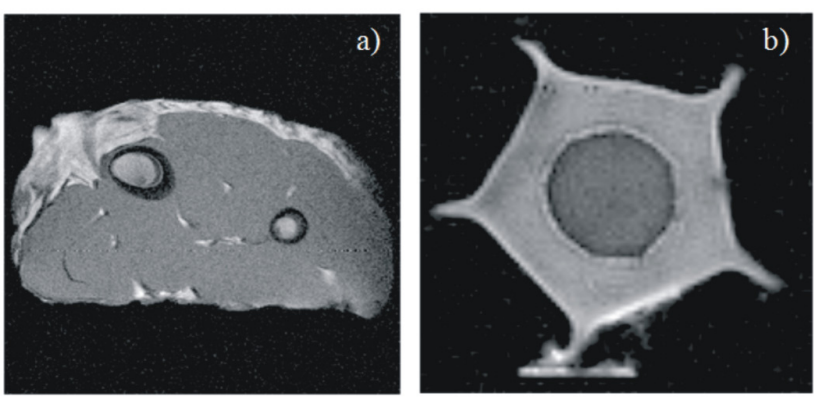

Fig.8. Spin-density MR imaging of biological tissue, a) chicken wing (SE method, $T_{\mathrm{E}} 17 \mathrm{~ms}, 40 \times 40 \mathrm{~mm}, 256 \times 256 \mathrm{px}$ ), b) euphorbia (SE method, $T_{\mathrm{E}}=17 \mathrm{~ms}, 30 \times 30 \mathrm{~mm}, 128 \times 128 \mathrm{px}$ ).

\section{RESULTS AND DISCUSSION}

Signal-to-noise ratio is the ratio of the mean value of image intensity in a selected space to the standard deviation of noise in the space outside of the image. It follows from the measurement that the signal-to-noise ratio obtained is roughly the same for all the coils. Table 1 gives the active resistances $R$ and inductances $L$ of all coils with inserted sample, measured on an RLC analyzer for the frequency $f=200 \mathrm{MHz}$. From the measurement it follows that the image noise is not due to the noise of the active resistance of RF coil but is given by the properties of the MR preamplifier. The inductances of slotted-tube and parallel-plate coils are markedly lower compared to the saddle coil. Both these coils can therefore be tuned at the $200 \mathrm{MHz}$ frequency even for larger dimensions and they can be used, for example, to measure small animals.

The homogeneous space for the parallel-plate coil on a circle of $20 \mathrm{~mm}$ in diameter is smaller than $\pm 10 \%$ of $B_{1}$. The inhomogeneous phase in a circular space of $20 \mathrm{~mm}$ in diameter is up to $10^{\circ}$. From both the experimental measurement and numerical modeling it is evident that for the measurement of small animals and biological objects the parallel-plate coil is of greater advantage than the saddle or slotted-tube coil.

\section{CONCLUSION}

The paper presents a comparison of the properties of RF coils of three configurations for MRI measurements on small animals. The two newly proposed configurations, modified slotted-tube and parallel-plate coils, have under identical homogeneity of field $B_{1}$ a larger exploitable application area (field of view). The two RF coils have the same SNR, which is due to the noise of RF pre-amplifier. Since the slotted-tube and parallel-plate coils have a lower inductance compared with the saddle coil, they can at large dimensions be tuned to resonance at the $200 \mathrm{MHz}$ frequency even if they are of larger dimensions. Results of the comparison indicate that the parallel-plate coil is of advantageous properties when measuring small animals.

\section{ACKNOWLEDGMENT}

The work described in the paper was financially supported by the research project GACR 13-09086S, research plan MSM 0021630513 and project of the BUT Grant Agency FEKTS-10-13.

\section{REFERENCES}

[1] Bogdanov, G., Ludwig, R. (2002). A coupled microstrip line transverse electromagnetic resonator model for high-field magnetic resonance imaging. Magnetic Resonance in Medicine, 47, 579-593.

[2] Akgun, C.E., DelaBarre, L., Sohn, S.M., Snyder, C., Adriany, G., Ugurbil, K., Vaughan, J.T., Gopinath, A. (2009). Novel multi-channel transmission line coil for high field magnetic resonance imaging. In IEEE MTTS International Microwave Symposium Digest, 7-12 June 2009. IEEE, 1425-1428.

[3] Benabdallah, N., Benahmed, N., Benyoucef, B., Bouhmidi, R., Khelif, M. (2007). Electromagnetic analysis of the slotted-tube resonator with a circular cross section for MRI applications. Physics in Medicine and Biology, 52, 4943-4952.

[4] Alderman, D.W., Grant, D.M. (1979). An efficient decoupler coil design which reduces heating in conductive samples in superconducting spectrometers. Journal of Magnetic Resonance, 34, 425-433.

[5] Li, S., Yang, Q.X., Smith, M.B. (1994). RF coil optimization: Evaluation of B1field homogeneity using field histograms and finite element calculations. Magnetic Resonance Imaging, 12, 1079-1087.

[6] Chen, Q., Sawaya, K., Uno, T., Adachi, S., Ochi, H., Yamamoto, E.A. (1994). Three dimensional analysis of slotted tube resonator for MRI. IEEE Transaction on Medical Imaging, 13 (4).

[7] Ben Ahmed, N., Feham, M. (2006). Analyzing EM parameters for shielded bandline. Microwaves \& $R F$, 45, 86-92.

[8] Ben Ahmed N., Feham, M., Khelif, M., (2006). Analysis and design of a coupled coaxial line TEM resonator for magnetic resonance imaging. Physics in Medicine and Biology, 51, 2093-2099.

[9] Hayes, C.E., Edelstein, W.A., Schenck, J.F., Muller, O.M., Eash, M.J. (1985). An efficient, highly homogeneous radiofrequency coil for whole-body NMR imaging at 1.5T. Journal of Magnetic Resonance, 64, 622-628.

[10] Baertlein, B.A., Ozbay, O., Ibrahim, T., Lee, R., Yu, Y., Kangarlu, A., Robitaille, P.M.L. (2000). The theoretical model for an MRI radiofrequency resonator. IEEE Transactions on Biomedical Engineering, 47, 535-546.

[11] Vaughan, T., DelaBarre, L., Snyder, C., Tian, J., Akgun, C., Shrivastava, D., Liu, W., Olson, C., Adriany, G., Strupp, J., Andersen, P., Gopinath, A., Van de Moortele, P-F., Garwood, M., Ugurbil, K. (2006). 9.4T human MRI: Preliminary results. Magnetic Resonance in Medicine, 56 (6), 1274-1282.

[12] Vaughan, J., DelaBarre, L., Snyder, C., Adriany, G., Collins, C., Van de Moortele, P-F., Ritter, J., Strupp, J., Andersen, P., Tian, J., Smith, M., Ugurbil, K. (2005). RF image optimization at $7 \mathrm{~T}$ and 9.4T. In International Society for Magnetic Resonance in Medicine, 13, 953. 
[13] Ibrahim, T., Lee, R., Robitaille, P. (2001). Effect of RF coil excitation on field inhomogeneity at ultra-high fields: A field optimized TEM resonator. Magnetic Resonance Imaging, 19, 1339-1347.

[14] Smirg, O., Liberda, O., Smekal, Z., Sprlakova-Pukova, A. (2012). A MRI slice segmentation and 3D modelling of temporomandibular joint measured by microscopic coil. Measurement Science Review, 12 (3), 74-81.

[15] Přibil, J., Gogola, D., Dermek, T., Frollo, I. (2012). Design, realization and experiments with a new RF head probe coil for human vocal tract imaging in an NMR device. Measurement Science Review, 12 (3), 98-103.

[16] Meadowcroft, M.D., Zhang, S., Liu, W., Park, B.S., Connor, J.R., Collins, C.M., Smith, M.B., Yang, Q.X. (2007). Direct magnetic resonance imaging of histological tissue samples at 3.0T. Magnetic Resonance in Medicine, 57 (5), 835-841.

[17] Marzola, P., Osculati, F., Sbarbati, A. (2003), High field MRI in preclinical research. European Journal of Radiology, 48 (2), 165-170.

[18] Vazquez, F., Martin, R., Marrufo, O., Rodriguez, A.O. (2010). Waveguide magnetic resonance imaging at 3 Tesla. In Joint Annual Meeting ISMRM-ESMRMB.
[19] Brunner, D.O., De Zanche, N., Froehlich, J., Paska, J., Pruessmann, K.P. (2009). Travelling-wave nuclear magnetic resonance. Nature, 457 (7232), 994-U2.

[20] Zhang, J., Balcom, B.J. (2010). Parallel-plate RF resonator imaging of chemical shift resolved capillary flow. Magnetic Resonance Imaging, 28, 826-833.

[21] Bobroff, S., McCarthy, M.J. (1999). Variations on the slotted-tube resonator: Rectangular and elliptical coils. Magnetic Resonance Imaging, 17 (5), 783-789.

[22] Sheen, J. (2008). A dielectric resonator method of measuring dielectric properties of low loss materials in the microwave region. Measurement Science and Technology, 19 (5), 055701.

[23] Akay, M.F., Prokopenko, Y., Kharkovsky, S. (2004) Resonance characteristics of whispering gallery modes in parallel-plates-type cylindrical dielectric resonators. Microwave and Optical Technology Letters, 40 (2), 96-101.

[24] Nespor, D., Bartusek, K., Fiala, P. (2011). Using numerical analysis for NMR coils optimization. PIERS Proceedings, 12-16 September 2011. The EM Academy, 72-75.

Received September 26, 2013. Accepted June 12, 2014. 